\title{
On Liu's Simplicial Depth and Randles' Interdirections
}

\author{
Robert Serfling ${ }^{\mathrm{a}, *}$, Yunfei Wang ${ }^{\mathrm{a}}$ \\ ${ }^{a}$ Department of Mathematical Sciences, University of Texas at Dallas, Richardson, Texas 75080, USA
}

\begin{abstract}
At about the same time (approximately 1989), R. Liu introduced the notion of simplicial depth and R. Randles the notion of interdirections. These completely independent and seemingly unrelated initiatives, serving different purposes in nonparametric multivariate analysis, have spawned significant activity within their quite different respective domains. A surprising and fruitful connection between the two notions is shown. Exploiting the connection, statistical procedures based on interdirections can be modified to use simplicial depth instead, at considerable reduction of computational burden in the case of dimensions 2,3 , and 4 . Implications regarding multivariate sign test statistics are discussed in detail, and several other potential applications are noted.
\end{abstract}

Keywords: Simplicial depth, Interdirections, Multivariate, Sign tests, Computational burden.

2010 MSC: Primary 62H99, Secondary 62G99

\section{Introduction}

In order to define affine equivariant order statistics and related quantities in the setting of multivariate data in higher dimensional space where there is no natural order, notions of depth function for the purpose of measuring centrality of points $\boldsymbol{x}$ in $\mathbb{R}^{d}$ relative to a given distribution $F$ on $\mathbb{R}^{d}$ have been developed, beginning with the seminal halfspace depth of Tukey (1975). When a quite different version, the simplicial depth, was introduced by Liu $(1988,1990)$, it became realized that in fact there are many viable and interesting implementations of the idea of depth function. Among the many versions of depth function that have been formulated, the most prominent in current practice are perhaps the halfspace, simplicial, Mahalanobis, spatial, and projection depths. A review of depth functions with respect to structural features is provided by Zuo and Serfling (2000a), and the wide range of their methodological applications is laid out by Liu, Parelius, and Singh (1999).

In general, a depth function induces a definition of multivariate median, given by the point(s) of maximal depth. Also, corresponding quantile and rank functions are generated by the nested contours of equal centrality at levels decreasing from the "center". Depth functions desirably are affine invariant. On the other hand, one may initially formulate some multivariate rank function and generate corresponding depth and quantile functions. For some discussion of such interrelations, see Serfling (2006a, 2010).

With either a depth function or a rank function in hand, one may formulate an associated multivariate "sign test" of a hypothesized "center" $\boldsymbol{\theta}_{0}$, either by evaluating the sample depth function at $\boldsymbol{\theta}_{0}$, or by evaluating the sample rank function at $\boldsymbol{\theta}_{0}$. When the depth and rank functions correspond to each other, these are equivalent. The "center" might be

\footnotetext{
* Corresponding author

URL: serfling@utdallas.edu (Robert Serfling), www.utdallas.edu/ ${ }^{\text {serfling (Robert Serfling) }}$
} 
formulated as the multivariate median according to a particular depth notion, or as the point of symmetry according to a given notion of symmetry. With respect to the latter, we will here refer to several key notions of symmetry, elliptical symmetry, central symmetry, elliptical directions, angular symmetry, and halfspace symmetry. See Randles (2000) and Serfling (2006b) for general discussion and the following relationships:

$$
\begin{aligned}
& \text { elliptical } \Longrightarrow \text { central } \Longrightarrow \text { angular } \Longrightarrow \text { halfspace, } \\
& \text { elliptical } \Longrightarrow \text { elliptical directions } \Longrightarrow \text { angular } \Longrightarrow \text { halfspace. }
\end{aligned}
$$

Of most interest for our purposes are central symmetry, denoting $\boldsymbol{X}-\boldsymbol{\theta}_{0} \stackrel{d}{=} \boldsymbol{\theta}_{0}-\boldsymbol{X}$, and angular (or "directional") symmetry, denoting $\boldsymbol{S}(\boldsymbol{X}-\boldsymbol{\theta}) \stackrel{d}{=} \boldsymbol{S}(\boldsymbol{\theta}-\boldsymbol{X})$, where $\stackrel{d}{=}$ denotes equality in distribution and $\boldsymbol{S}(\cdot)$ is the spatial sign function (or unit vector function),

$$
\boldsymbol{S}(\boldsymbol{x})=\left\{\begin{array}{cc}
\frac{\boldsymbol{x}}{\|\boldsymbol{x}\|}, & \boldsymbol{x} \neq \mathbf{0} \\
\mathbf{0}, & \boldsymbol{x}=\mathbf{0}
\end{array}\right.
$$

for $\boldsymbol{x} \in \mathbb{R}^{d}$, with $\|\cdot\|$ the usual Euclidean norm.

Our goal here is to establish and exploit a special relationship between the simplicial depth of Liu $(1988,1990)$ and the notion of "interdirections" introduced by Randles (1989) in formulating a new multivariate sign test. Specifically, we introduce an "interdirections depth" and show that it is linearly equivalent to the simplicial depth in dimension $d=2$ and approximately equivalent in higher dimension $d \geq 3$. In their direct formulations, the simplicial depth and the interdirections both pose a computational burden of order $O\left(n^{d+1}\right)$, which becomes quickly prohibitive as $d$ increases. However, for the simplicial depth, more favorable algorithms have been developed in the cases $d=2,3$, and 4 . Also, the simplicial depth is conceptually simpler than the interdirections. Statistical procedures based on interdirections can thus be modified to use simplicial depth instead, thereby taking advantage of more efficient computational algorithms and greater conceptual appeal, without loss of practical effectiveness. In particular, we compare the sign test introduced by Liu (1990) based on simplicial depth and the above-mentioned sign test of Randles (1989).

We proceed as follows. The simplicial depth is reviewed in Section 2, along with details on its computation. The interdirections and our associated interdirections depth function are treated in Section 3. The special connection between the two depths is developed in Section 4, where also comparisons with respect to computation time are made. Several statistical procedures that use interdirections and which could profitably be modified to use simplicial depth instead are noted in Section 5, with selected multivariate sign tests for location treated in detail.

\section{Liu's simplicial depth}

\subsection{Formulation and properties}

Let us denote by $S\left[\boldsymbol{x}_{1}, \ldots, \boldsymbol{x}_{d+1}\right]$ the closed simplex in $\mathbb{R}^{d}$ with vertices $\boldsymbol{x}_{1}, \ldots, \boldsymbol{x}_{d+1}$. (If the vertices are not affinely independent, then $S\left[\boldsymbol{x}_{1}, \ldots, \boldsymbol{x}_{d+1}\right]$ is actually not a simplex but rather a convex object contained within a $k$-flat for some $k<d$.) Relative to a given distribution $F$ on $\mathbb{R}^{d}$, Liu $(1988,1990)$ introduced the simplicial depth,

$$
D_{S}(\boldsymbol{x}, F)=P_{F}\left(\boldsymbol{x} \in S\left[\boldsymbol{X}_{1}, \ldots, \boldsymbol{X}_{d+1}\right]\right), \boldsymbol{x} \in \mathbb{R}^{d},
$$

which associates with a point $\boldsymbol{x}$ the $F$-probability that it is covered by a random simplex whose vertices are given by $d+1$ independent hypothetical observations $\boldsymbol{X}_{1}, \ldots, \boldsymbol{X}_{d+1}$ from $F$. (For continuous $F$, these are affinely independent, with probability 1.) Besides its evident 
simplicity and conceptual appeal, the simplicial depth is easily seen to be affine invariant. The point $\boldsymbol{M}_{F}$ of maximal simplicial depth defines the simplicial median.

Two important properties of $D_{S}(\boldsymbol{x}, F)$ established by Liu (1990) in the case that $F$ is absolutely continuous on $\mathbb{R}^{d}$ and angularly symmetric sbout $\boldsymbol{M}_{F}$ are:

(a) $D_{S}(\boldsymbol{x}, F)$ is monotone nonincreasing along rays outward from $\boldsymbol{\theta}$,

(b) $D_{S}\left(\boldsymbol{M}_{F}, F\right)=2^{-d}$.

These properties together imply that $D_{S}(\boldsymbol{x}, F) \leq 2^{-d}, \boldsymbol{x} \in \mathbb{R}^{d}$.

For a sample $\mathbb{X}_{n}=\left\{\boldsymbol{X}_{1}, \ldots, \boldsymbol{X}_{n}\right\}$ from $F$, the sample simplicial depth is given by

$$
D_{S}\left(\boldsymbol{x}, \mathbb{X}_{n}\right)=\left(\begin{array}{c}
n \\
d+1
\end{array}\right)^{-1} \sum_{1 \leq i_{1}<\cdots<i_{d+1} \leq n} \mathbf{1}\left\{\boldsymbol{x} \in S\left[\boldsymbol{X}_{i_{1}}, \ldots, \boldsymbol{X}_{i_{d+1}}\right]\right\}, \boldsymbol{x} \in \mathbb{R}^{d}
$$

where $\mathbf{1}\{A\}$ denotes the indicator function of the event $A$. Note that, for each fixed $\boldsymbol{x}$, the sample version is precisely a $U$-statistic, based on the kernel

$$
h_{S}\left(\boldsymbol{x} ; \boldsymbol{x}_{1}, \ldots, \boldsymbol{x}_{d+1}\right)=\mathbf{1}\left\{\boldsymbol{x} \in S\left[\boldsymbol{x}_{1}, \ldots, \boldsymbol{x}_{d+1}\right]\right\} .
$$

We denote by $\boldsymbol{M}_{n}$ the sample simplicial median.

\subsection{Asymptotics}

Asymptotic results for the sample simplicial depth and sample simplicial median are available assuming merely angular symmetry of $F$. Liu (1990) shows consistency of $D_{S}\left(\boldsymbol{x}, \mathbb{X}_{n}\right)$ for estimation of $D_{S}(\boldsymbol{x}, F)$, and Dümbgen (1992) and Arcones and Giné (1993) develop asymptotic distribution theory for the random function $\left\{D_{S}\left(\boldsymbol{x}, \mathbb{X}_{n}\right), \boldsymbol{x} \in \mathbb{R}^{d}\right\}$ considered as a special case of U-process. In particular, for $\boldsymbol{x} \neq \boldsymbol{\theta}, D_{S}\left(\boldsymbol{x}, \mathbb{X}_{n}\right)$ is a nondegenerate Ustatistic with normal limit distribution. However, as remarked by Liu (1990), $D_{S}\left(\boldsymbol{M}_{F}, \mathbb{X}_{n}\right)$ is a degenerate U-statistic with limit distribution a weighted sum of independent chi-square variables. Relevant asymptotic theory for $\boldsymbol{M}_{n}$ is developed by Arcones, Chen and Giné (1994) and de la Peña and Ginè (1999):

$$
n^{1 / 2}\left(\boldsymbol{M}_{n}-\boldsymbol{M}_{F}\right) \stackrel{d}{\rightarrow} N\left(\mathbf{0}, \boldsymbol{\Gamma}_{F}\right), n \rightarrow \infty,
$$

for a suitable covariance matrix $\boldsymbol{\Gamma}_{F}$ depending on $F$. (For $F$ standard bivariate normal, $\boldsymbol{\Gamma}_{F}$ $=\frac{4}{\pi} \boldsymbol{I}_{2}$, showing that the simplicial median has asymptotic relative efficiency $4 / \pi=0.785$ relative to the sample mean, in dimension $d=2$.)

\subsection{Sign and median tests}

As discussed by Liu (1990), applying properties (a) and (b), a test of the hypothesis that $F$ is angularly symmetric about $\boldsymbol{\theta}_{0}$ may be carried out in terms of the test statistic

$$
S_{n}=2^{-d}-D_{S}\left(\boldsymbol{\theta}_{0}, \mathbb{X}_{n}\right) .
$$

Under $H_{0}, S_{n}$ is a nondegenerate U-statistic as noted above. Alternatively, and equivalently, assuming angular symmetry of $F$, one may test the hypothesis $H_{0}: \boldsymbol{M}_{F}=\boldsymbol{\theta}_{0}$ using the test statistic based on $\boldsymbol{M}_{n}$ and its asymptotic normality. However, as noted below, the

computational cost of $\boldsymbol{M}_{n}$ is much higher than that of $S_{n}$. We further discuss these tests, in comparison with others, in Section 5.1. 


\subsection{Computational considerations}

For efficient computation of the sample simplicial depth, key results are reviewed in Burr, Rafalin, and Souvaine (2006), who also treat variations on the definition of simplicial depth. In particular, for $d=2$, the simplicial depth of a single point $\boldsymbol{x}$ (e.g., $\boldsymbol{\theta}_{0}$ ) can be computed in $O(n \log n)$ time and that of all $n$ data points in $O\left(n^{2}\right)$ time (see Khuller and Mitchell, 1990, Gil, Steiger, and Wigderson, 1992, Rousseeuw and Ruts, 1996, and Aloupis et al, 2001). In dimensions $d=3$ and $4, O\left(n^{2}\right)$ and $O\left(n^{4}\right)$ algorithms are available, respectively, for computation of the simplicial depth of a single point (see Khuller and Mitchell, 1990, and Gil, Steiger, and Wigderson, 1992). However, for $d \geq 5$, no algorithms have been developed faster than simply generating all simplices and counting the ones enclosing the given point, using $O\left(n^{d+1}\right)$ time.

In the case of higher dimension, a standard approach toward overcoming computational complexity is to take just a random subset of the data. Another possibility is to compute merely the "simplicial depth matrix"

$$
\boldsymbol{D}_{S}(\boldsymbol{x}, \mathbb{X})=\left[D_{S}\left(\left(x_{i}, x_{j}\right), \mathbb{X}_{n}^{(i, j)}\right)\right]_{d \times d}
$$

of bivariate simplicial depths taken pairwise over the components of $\boldsymbol{x}=\left(x_{1}, \ldots, x_{d}\right)^{\prime}$, which can be computed in $O(n \log n)$ time for any fixed dimension $d$. These possibilities will be explored in a separate study.

Computation of the simplicial median is considerably more challenging and costly than the simplicial depth. For $d=2$, Aloupis et al (2003) provide algorithms that entail querying all $O\left(n^{4}\right)$ intersections of the $O\left(n^{2}\right)$ lines formed by pairs of data points. One algorithm uses $O\left(n^{4}\right)$ time and space, the other $O\left(n^{4} \log n\right)$ time and $O\left(n^{2}\right)$ space. Boros and Füredi (1984) establish a worst case order of $O\left(n^{3}\right)$ for computation of the bivariate simplicial median. For comparison, the best algorithm for computation of the bivariate halfspace median requires only $O\left(n \log ^{3} n\right)$ time (Langerman and Steiger, 2003). Of course, as a surrogate for the sample simplicial median, the deepest sample data point with respect to simplicial depth can be found in $O\left(n^{2} \log n\right)$ time and is the version given in the $\mathrm{R}$ package depth. We note, however, that the deepest sample data point has a different limit distribution than the actual sample simplicial median.

\section{Randles' interdirections}

For a distribution $F$ on $\mathbb{R}^{d}$ assumed to be angularly symmetric about $\boldsymbol{\theta}$ in $\mathbb{R}^{d}$, Randles (1989) considers the hypothesis $H_{0}: \boldsymbol{\theta}=\boldsymbol{\theta}_{0}$ and develops a type of "sign test" based on "interdirections" defined as follows relative to a sample $\mathbb{X}_{n}=\left\{\boldsymbol{X}_{1}, \ldots, \boldsymbol{X}_{n}\right\}$. For each pair $\{i, j\}, 1 \leq i \neq j \leq n$, consider the $\left(\begin{array}{l}n-2 \\ d-1\end{array}\right)$ hyperplanes formed by $\boldsymbol{\theta}_{0}$ with any $d-1$ points in $\mathbb{X}_{n}$ other than $\boldsymbol{X}_{i}$ and $\boldsymbol{X}_{j}$. The count $C_{i j}=C_{j i}$ of those hyperplanes for which $\boldsymbol{X}_{i}-\boldsymbol{\theta}_{0}$ and $\boldsymbol{X}_{j}-\boldsymbol{\theta}_{0}$ are on opposite sides measures the angular distance between $\boldsymbol{X}_{i}-\boldsymbol{\theta}_{0}$ and $\boldsymbol{X}_{j}-\boldsymbol{\theta}_{0}$, relative to the positions of the other points. In effect, it counts the number of points lying between $\boldsymbol{X}_{i}-\boldsymbol{\theta}_{0}$ and $\boldsymbol{X}_{j}-\boldsymbol{\theta}_{0}$, just as in the univariate case the number of points between two points is given by the difference of their ranks. The counts $\left\{C_{i j}\right\}$ are called interdirections and are invariant under nonsingular linear transformations.

Randles converts the counts to equivalent fractions, with two variants. These are, for $1 \leq i \neq j \leq n$,

$$
\tilde{f}_{i j}=C_{i j} /\left(\begin{array}{l}
n-2 \\
d-1
\end{array}\right)
$$


which is natural and straightforward, being based on the number of hyperplanes involved in the count $C_{i j}$, and

$$
f_{i j}= \begin{cases}{\left[C_{i j}+d_{n}\right] /\left(\begin{array}{c}
n \\
d-1
\end{array}\right)} & \text { if } i \neq j, \\
0 & \text { if } i=j,\end{cases}
$$

which, with $d_{n}=\left[\left(\begin{array}{c}n \\ d-1\end{array}\right)-\left(\begin{array}{c}n-2 \\ d-1\end{array}\right)\right] / 2$, is the fraction of all $\left(\begin{array}{c}n \\ d-1\end{array}\right)$ data-based hyperplanes which fall between $\boldsymbol{X}_{i}-\boldsymbol{\theta}_{0}$ and $\boldsymbol{X}_{j}-\boldsymbol{\theta}_{0}$, counting $1 / 2$ whenever one or both of the pair $\boldsymbol{X}_{i}-\boldsymbol{\theta}_{0}$ and $\boldsymbol{X}_{j}-\boldsymbol{\theta}_{0}$ fall directly on the hyperplane. Relative to the plane formed by $\boldsymbol{\theta}_{0}, \boldsymbol{X}_{i}-\boldsymbol{\theta}_{0}$, and $\boldsymbol{X}_{j}-\boldsymbol{\theta}_{0}$, the quantity $\pi f_{i j}$ estimates the angle between $\boldsymbol{X}_{i}-\boldsymbol{\theta}_{0}$ and $\boldsymbol{X}_{j}-\boldsymbol{\theta}_{0}$.

\subsection{Randles' interdirections sign test}

In formulating an associated sign test statistic, Randles applies the cosine function to the quantities $\left\{f_{i j}\right\}$ and forms the sign test statistic $V_{n}=\frac{d}{n} \sum_{i=1}^{n} \sum_{j=1}^{n} \cos \left(\pi f_{i j}\right)$, with $H_{0}$ rejected for high values. For $F$ having elliptical directions, Randles shows that $V_{n}$ has under $H_{0}$ a limiting $\chi_{d}^{2}$ distribution. For $d=2, V_{n}$ reduces to the bivariate sign test of Blumen (1958). We further discuss these tests in Section 5.1.

\subsection{Computational considerations}

Note that, for each of the $\left(\begin{array}{l}n \\ 2\end{array}\right)$ choices of pair $(i, j)$, the computation of $C_{i j}$ entails $\left(\begin{array}{l}n-2 \\ d-1\end{array}\right)$ sets of $d-1$ data points. Thus computation of the full set of $\left\{C_{i j}, 1 \leq i<j \leq n\right\}$ of interdirections treats

$$
N(n, d)=\left(\begin{array}{l}
n \\
2
\end{array}\right)\left(\begin{array}{l}
n-2 \\
d-1
\end{array}\right)=O\left(n^{d+1}\right)
$$

different sets of size $d+1$. Even for $d=2$, this is prohibitive for large $n$. As discussed in Hallin and Paindaveine (2002a), this computational burden motivates the use of other test statistics that are more readily computed.

\subsection{Interdirections depth}

As mentioned in Section 1, not only do depth functions induce rank functions, but the converse holds. Thus, although the interdirections have been introduced strictly in the context of sign tests and related tests, it is important to explore whether there is an associated notion of "median", just as in the univariate case the sign test is associated with estimation of a median. More comprehensively, we ask whether we can identify a productive "interdirections depth function". Indeed, we now formulate and study such a depth function.

Note that for $\boldsymbol{\theta}_{0}$ near the center of the data cloud, the corresponding counts $C_{i j}$ tend to be high, whereas they tend to be low for $\boldsymbol{\theta}_{0}$ near the margin of the data cloud or far away from it. That is, $C_{i j}=C_{i j}\left(\boldsymbol{\theta}_{0}, \mathbb{X}_{n}\right)$ measures the centrality of $\boldsymbol{\theta}_{0}$ relative to the locations of $\boldsymbol{X}_{i}$ and $\boldsymbol{X}_{j}$ in the full data set $\mathbb{X}_{n}$. Accordingly, we analogously define $C_{i j}\left(\boldsymbol{x}, \mathbb{X}_{n}\right)$ for all points $\boldsymbol{x}$ in $\mathbb{R}^{d}$, and also equivalently convert to the associated fractions $\tilde{f}_{i j}\left(\boldsymbol{x}, \mathbb{X}_{n}\right)=C_{i j}\left(\boldsymbol{x}, \mathbb{X}_{n}\right) /\left(\begin{array}{c}n-2 \\ d-1\end{array}\right)$. (Alternatively, the fractions $f_{i j}$ could be used, or even the quantities $\cos \left(\pi f_{i j}\right)$, but we prefer the $\widetilde{f}_{i j}$, which capture quite directly and naturally the "interdirections information" regarding centrality of points.) On this basis, we define the (sample) interdirections depth function by

$$
D_{I}\left(\boldsymbol{x}, \mathbb{X}_{n}\right)=\left(\begin{array}{l}
n \\
2
\end{array}\right)^{-1} \sum_{1 \leq i<j \leq n} \widetilde{f}_{i j}\left(\boldsymbol{x}, \mathbb{X}_{n}\right), \boldsymbol{x} \in \mathbb{R}^{d},
$$

taking values in $[0,1]$. This provides a fuller way to apply the interdirections. Later we will see that the population version of $D_{I}\left(\boldsymbol{x}, \mathbb{X}_{n}\right)$, given by taking the expectation, can be carried 
out by taking the expectation of a somewhat simpler quantity than $\widetilde{f}_{i j}\left(\boldsymbol{x}, \mathbb{X}_{n}\right)$, which is a complex function of all the data points in $\mathbb{X}_{n}$.

Very importantly, the interdirections depth function has a representation (pointwise) as a $U$-statistic, developed as follows. Note that each term generating the count $C_{i j}\left(\boldsymbol{x}, \mathbb{X}_{n}\right)$ involves $d+1$ points of $\mathbb{X}_{n}$. Instead of organizing the count information separately by pairs $\{i, j\}$, we instead organize it by the separate subsets of $\mathbb{X}_{n}$ of size $d+1$. For this purpose, we first introduce, for each $\{i, j\}$, the functions $g_{i j}\left(\boldsymbol{x} ; \boldsymbol{x}_{1}, \ldots, \boldsymbol{x}_{d+1}\right)=1$ or 0 according as the hyperplane formed by $\boldsymbol{x}$ and $\left\{\boldsymbol{x}_{k}: k \neq i, k \neq j, 1 \leq k \leq d+1\right\}$ separates $\boldsymbol{x}_{i}$ and $\boldsymbol{x}_{j}$, or not. Then we average these functions over all pairs $\{i \neq j\}$ to form the kernel

$$
h_{I}\left(\boldsymbol{x} ; \boldsymbol{x}_{1}, \ldots, \boldsymbol{x}_{d+1}\right)=\left(\begin{array}{c}
d+1 \\
2
\end{array}\right)^{-1} \sum_{1 \leq i<j \leq d+1} g_{i j}\left(\boldsymbol{x} ; \boldsymbol{x}_{1}, \ldots, \boldsymbol{x}_{d+1}\right) .
$$

It then readily follows that

$$
D_{I}\left(\boldsymbol{x}, \mathbb{X}_{n}\right)=\left(\begin{array}{c}
n \\
d+1
\end{array}\right)^{-1} \sum_{1 \leq i_{1}<\cdots<i_{d+1} \leq n} h_{I}\left(\boldsymbol{x} ; \boldsymbol{X}_{i_{1}}, \ldots, \boldsymbol{X}_{i_{d+1}}\right),
$$

expressing $D_{I}\left(\boldsymbol{x}, \mathbb{X}_{n}\right)$ as precisely a U-statistic with kernel $h_{I}\left(\boldsymbol{x} ; \boldsymbol{x}_{1}, \ldots, \boldsymbol{x}_{d+1}\right)$. (Note that (4) can be reexpressed as $N(n, d)=\left(\begin{array}{c}n \\ d+1\end{array}\right)\left(\begin{array}{c}d+1 \\ 2\end{array}\right)$.) Thus the asymptotic behavior of this depth function follows using standard U-statistic theory. The computation of the interdirections depth at a single point requires, as noted above for computation of the interdirections, $O\left(n^{d+1}\right)$ time. Finally, we note that the population interdirections depth $D(\boldsymbol{x}, F)$ is given simply by taking expectations in the U-statistic representation (7), yielding $D_{I}(\boldsymbol{x}, F)=$ $E h_{I}\left(\boldsymbol{x} ; \boldsymbol{X}_{1}, \ldots, \boldsymbol{X}_{d+1}\right), \boldsymbol{x} \in \mathbb{R}^{d}$, or equivalently $D_{I}(\boldsymbol{x}, F)=E g_{12}\left(\boldsymbol{x} ; \boldsymbol{X}_{1}, \ldots, \boldsymbol{X}_{d+1}\right), \boldsymbol{x} \in$ $\mathbb{R}^{d}$. In the case $d=2$, a further simplification holds using the connection developed in the following section.

\section{Connection between $D_{I}$ and $D_{S}$}

We show that the simplicial and interdirections depth functions are exactly equivalent in the bivariate case and nearly so in higher dimensions. Of course, taking computational burden into consideration, the bivariate case is the one of greatest practical significance.

Note that, for $F$ continuous on $\mathbb{R}^{d}$, it holds with probability 1 that each simplex $S\left[\boldsymbol{X}_{i_{1}}, \ldots, \boldsymbol{X}_{i_{d+1}}\right]$ formed from $\mathbb{X}_{n}$ does not lie in any lower-dimensional hyperplane. That is, with probability $1, \mathbb{X}_{n}$ is in "general position". Also, for $F$ continuous, the union $H\left(\mathbb{X}_{n}\right)$ of the hyperplanes corresponding to all the faces of all the simplices $S\left[\boldsymbol{X}_{i_{1}}, \ldots, \boldsymbol{X}_{i_{d+1}}\right]$ has probability 0 . Therefore, for convenience and simplicity, we assume that $\mathbb{X}_{n}$ is in general position and ignore points in $H\left(\mathbb{X}_{n}\right)$, which is justified with probability 1 for $F$ continuous. Although, therefore, the data points themselves are excluded in our formal relationships between $D_{I}\left(\boldsymbol{x}, \mathbb{X}_{n}\right)$ and $D_{S}\left(\boldsymbol{x}, \mathbb{X}_{n}\right)$, this is of little or no consequence in the applications of our results.

Theorem 1. Consider the case $d=2$ and assume that $\mathbb{X}_{n}$ is in general position. Then the sample interdirections and simplicial depth functions are linearly equivalent on $\mathbb{R}^{2} \backslash H\left(\mathbb{X}_{n}\right)$ :

$$
D_{I}\left(\boldsymbol{x}, \mathbb{X}_{n}\right)=\frac{2}{3} D_{S}\left(\boldsymbol{x}, \mathbb{X}_{n}\right)+\frac{1}{3}, \boldsymbol{x} \in \mathbb{R}^{2} \backslash H\left(\mathbb{X}_{n}\right) .
$$

Proof. We show that the corresponding kernels $h_{I}$ and $h_{S}$ in the respective U-statistic representations of $D_{I}$ and $D_{S}$ satisfy the same equivalence as in (8), i.e., that

$$
h_{I}=\mathbf{1}\left\{h_{S}=1\right\}+\frac{1}{3} \mathbf{1}\left\{h_{S}=0\right\}=\frac{2}{3} h_{S}+\frac{1}{3},
$$


subject to $\boldsymbol{x} \notin H\left(\mathbb{X}_{n}\right)$. Then (8) immediately follows via (7).

To extablish (9), let $\boldsymbol{x} \in \mathbb{R}^{2}$ and take any triplet from $\mathbb{X}_{n}$, say $\left\{\boldsymbol{X}_{1}, \boldsymbol{X}_{2}, \boldsymbol{X}_{3}\right\}$. We have

$$
h_{I}\left(\boldsymbol{x} ; \boldsymbol{X}_{1}, \boldsymbol{X}_{2}, \boldsymbol{X}_{3}\right)=\frac{g_{12}\left(\boldsymbol{x} ; \boldsymbol{X}_{1}, \boldsymbol{X}_{2}, \boldsymbol{X}_{3}\right)+g_{13}\left(\boldsymbol{x} ; \boldsymbol{X}_{1}, \boldsymbol{X}_{2}, \boldsymbol{X}_{3}\right)+g_{23}\left(\boldsymbol{x} ; \boldsymbol{X}_{1}, \boldsymbol{X}_{2}, \boldsymbol{X}_{3}\right)}{3} .
$$

Let us now consider the open planar region $A=S\left[\boldsymbol{X}_{1}, \boldsymbol{X}_{2}, \boldsymbol{X}_{3}\right]^{\circ}$ (the interior of $S$ ), the six open regions $B, C, D, E, F$, and $G$ outside of $A$ defined by extending the three edges of the simplex $S$, and six representative points $\boldsymbol{x}_{a}-\boldsymbol{x}_{g}$ within these regions, respectively. See Figure 1 for illustration.

First, let us consider $\boldsymbol{x} \in A$ (in which case $h_{S}\left(\boldsymbol{x} ; \boldsymbol{X}_{1}, \boldsymbol{X}_{2}, \boldsymbol{X}_{3}\right)=1$ ). In particular, let $\boldsymbol{x}=\boldsymbol{x}_{a}$. It is immediate that $g_{12}\left(\boldsymbol{x}_{a} ; \boldsymbol{X}_{1}, \boldsymbol{X}_{2}, \boldsymbol{X}_{3}\right)=1$ because the extended line $\boldsymbol{x}_{a} \boldsymbol{X}_{3}$ intersects the edge $\boldsymbol{X}_{1} \boldsymbol{X}_{2}$ and thus separates $\boldsymbol{X}_{1}$ and $\boldsymbol{X}_{2}$. Similarly, $g_{13}\left(\boldsymbol{x}_{a} ; \boldsymbol{X}_{1}, \boldsymbol{X}_{2}, \boldsymbol{X}_{3}\right)$ $=1=g_{23}\left(\boldsymbol{x}_{a} ; \boldsymbol{X}_{1}, \boldsymbol{X}_{2}, \boldsymbol{X}_{3}\right)$. Thus $h_{I}\left(\boldsymbol{x}_{a} ; \boldsymbol{X}_{1}, \boldsymbol{X}_{2}, \boldsymbol{X}_{3}\right)=1$ and we have

$$
h_{I}\left(\boldsymbol{x} ; \boldsymbol{X}_{1}, \boldsymbol{X}_{2}, \boldsymbol{X}_{3}\right)=1=h_{S}\left(\boldsymbol{x} ; \boldsymbol{X}_{1}, \boldsymbol{X}_{2}, \boldsymbol{X}_{3}\right), \boldsymbol{x} \in A .
$$

For $\boldsymbol{x}=\boldsymbol{x}_{b} \in B$, we readily see that $g_{12}\left(\boldsymbol{x}_{b} ; \boldsymbol{X}_{1}, \boldsymbol{X}_{2}, \boldsymbol{X}_{3}\right)=1$, but now $g_{13}\left(\boldsymbol{x}_{b} ; \boldsymbol{X}_{1}, \boldsymbol{X}_{2}, \boldsymbol{X}_{3}\right)$ $=0=g_{23}\left(\boldsymbol{x}_{b} ; \boldsymbol{X}_{1}, \boldsymbol{X}_{2}, \boldsymbol{X}_{3}\right)$, yielding $h_{I}\left(\boldsymbol{x}_{b} ; \boldsymbol{X}_{1}, \boldsymbol{X}_{2}, \boldsymbol{X}_{3}\right)=1 / 3$. In similar fashion, we also obtain $h_{I}\left(\boldsymbol{x} ; \boldsymbol{X}_{1}, \boldsymbol{X}_{2}, \boldsymbol{X}_{3}\right)=1 / 3$ for each of $\boldsymbol{x}=\boldsymbol{x}_{c}, \boldsymbol{x}_{d}, \boldsymbol{x}_{e}, \boldsymbol{x}_{f}$, and $\boldsymbol{x}_{g}$. Thus we have

$$
h_{I}\left(\boldsymbol{x} ; \boldsymbol{X}_{1}, \boldsymbol{X}_{2}, \boldsymbol{X}_{3}\right)=1 / 3 \text {, and } h_{S}\left(\boldsymbol{x} ; \boldsymbol{X}_{1}, \boldsymbol{X}_{2}, \boldsymbol{X}_{3}\right)=1, \boldsymbol{x} \notin A \text {. }
$$

We ignore points $\boldsymbol{x}$ on the extended lines defining the edges of $S\left[\boldsymbol{X}_{1}, \boldsymbol{X}_{2}, \boldsymbol{X}_{3}\right]$, which belong to $H\left(\mathbb{X}_{n}\right)$. Thus (9) holds, establishing (8).

Thus, for $d=2$, the interdirections and simplicial depths $D_{I}$ and $D_{S}$ are averages of equivalent indicator function kernels $h_{I}$ and $h_{S}$, each assigning one of two constants according as $\boldsymbol{x}$ does or does not belong to a pertinent set (the same set for each kernel). In particular, both kernels assign "1" for membership, whereas for nonmembership the simplicial kernel assigns " 0 " while the interdirections kernal assigns " $1 / 3$ ". Thus, in the bivariate case, the depths $D_{I}$ and $D_{S}$ are interchangeable for practical purposes.

For dimension $d \geq 3$, the situation is similar, although in a somewhat looser sense. The following result shows a "quasi-linear" equivalence of $D_{I}$ and $D_{S}$.

Theorem 2. Consider the case $d \geq 3$ and assume that $\mathbb{X}_{n}$ is in general position. Then the sample interdirections and simplicial depth functions are "quasi-linearly equivalent" on $\mathbb{R}^{d} \backslash H\left(\mathbb{X}_{n}\right)$, as follows:

$$
D_{I}\left(\boldsymbol{x}, \mathbb{X}_{n}\right)=\left(1-\frac{\alpha\left(\boldsymbol{x}, \mathbb{X}_{n}\right)}{\left(\begin{array}{c}
d+1 \\
2
\end{array}\right)}\right) D_{S}\left(\boldsymbol{x}, \mathbb{X}_{n}\right)+\frac{\alpha\left(\boldsymbol{x}, \mathbb{X}_{n}\right)}{\left(\begin{array}{c}
d+1 \\
2
\end{array}\right)}, \boldsymbol{x} \in \mathbb{R}^{d} \backslash H\left(\mathbb{X}_{n}\right),
$$

where $\alpha\left(\boldsymbol{x}, \mathbb{X}_{n}\right)$ is the average, over all $\left\{\boldsymbol{X}_{i_{1}}, \ldots, \boldsymbol{X}_{i_{d+1}}\right\}$ such that $\boldsymbol{x} \notin S\left[\boldsymbol{X}_{i_{1}}, \ldots, \boldsymbol{X}_{i_{d+1}}\right]$, of a function $\alpha\left(\boldsymbol{x}, \boldsymbol{X}_{i_{1}}, \ldots, \boldsymbol{X}_{i_{d+1}}\right)$ which is integer-valued, depends on the location of $\boldsymbol{x}$ relative to $S\left[\boldsymbol{X}_{i_{1}}, \ldots, \boldsymbol{X}_{i_{d+1}}\right]$, and satisfies

$$
2 \leq \alpha\left(\boldsymbol{x}, \boldsymbol{X}_{i_{1}}, \ldots, \boldsymbol{X}_{i_{d+1}}\right) \leq\left(\begin{array}{l}
d \\
2
\end{array}\right)
$$

with the lower bound sharp for $d=3$ and the upper bound sharp for all $d \geq 3$.

Proof. Again it suffices to treat the kernels $h_{I}$ and $h_{S}$ in the U-statistic representations. Consider any $(d+1)$-set from $\mathbb{X}_{n}$, say $\left\{\boldsymbol{X}_{1}, \ldots, \boldsymbol{X}_{d+1}\right\}$. We treat two cases.

Case 1: $\boldsymbol{x} \in S\left[\boldsymbol{X}_{1}, \ldots, \boldsymbol{X}_{d+1}\right]^{\circ}$. 
It readily is seen that $g_{i j}\left(\boldsymbol{x} ; \boldsymbol{X}_{1}, \ldots, \boldsymbol{X}_{d+1}\right)=1$ for all $1 \leq i<j \leq d+1$ and hence that

$$
h_{I}\left(\boldsymbol{x} ; \boldsymbol{X}_{1}, \ldots, \boldsymbol{X}_{d+1}\right)=1=h_{S}\left(\boldsymbol{x} ; \boldsymbol{X}_{1}, \ldots, \boldsymbol{X}_{d+1}\right)
$$

exactly as in the case $d=2$. Therefore, $h_{I}=1$ whenever $h_{S}=1$.

Case 2: $\boldsymbol{x} \notin S\left[\boldsymbol{X}_{1}, \ldots, \boldsymbol{X}_{d+1}\right]^{\circ}$.

Denote by $F_{i}, 1 \leq i \leq d+1$, the $\left(\begin{array}{c}d+1 \\ d\end{array}\right)=d+1$ faces of $S\left[\boldsymbol{X}_{1}, \ldots, \boldsymbol{X}_{d+1}\right]$. The following notation will be convenient:

$$
\begin{aligned}
H\left(F_{i}\right) & =\text { hyperplane of } F_{i} \\
H_{i} & =\text { open halfspace associated with } H\left(F_{i}\right) \text { in } S\left[\boldsymbol{X}_{1}, \ldots, \boldsymbol{X}_{d+1}\right]^{c}, \\
\boldsymbol{v}_{i} & =\text { the vertex of } S\left[\boldsymbol{X}_{1}, \ldots, \boldsymbol{X}_{d+1}\right] \text { not in } F_{i}, \\
A_{i} & =\left\{\boldsymbol{x} \in H_{i} \text { : line } \overline{\boldsymbol{x} \boldsymbol{v}_{i}} \text { passes through } F_{i}\right\} \\
B_{i j} & =H_{i} \cap H_{j}
\end{aligned}
$$

and

$\alpha\left(\boldsymbol{x}, \boldsymbol{X}_{1}, \ldots, \boldsymbol{X}_{d+1}\right)=$ number of pairs $\{i, j\}$ for which $g_{i j}\left(\boldsymbol{x} ; \boldsymbol{X}_{1}, \ldots, \boldsymbol{X}_{d+1}\right)=1$.

Case 2(a): $\boldsymbol{x} \in \bigcup_{i=1}^{d+1} A_{i}$.

Note that the $A_{i}$ are disjoint. Fix $i$ and select $\boldsymbol{x} \in A_{i}$.

(i) Consider any hyperplane $H$ formed by $\boldsymbol{x}, \boldsymbol{v}_{i}$, and any $d-2$ vertices from $F_{i}$. Each such $H$ intersects $S\left[\boldsymbol{X}_{1}, \ldots, \boldsymbol{X}_{d+1}\right]^{\circ}$ and separates the 2 vertices of $F_{i}$ not in $H$. Since there are $\left(\begin{array}{c}d \\ d-2\end{array}\right)=\left(\begin{array}{l}d \\ 2\end{array}\right)$ choices of such $H$, the resulting increment to $\alpha\left(\boldsymbol{x}, \boldsymbol{X}_{1}, \ldots, \boldsymbol{X}_{d+1}\right)$ is

$$
\text { increment }=\left(\begin{array}{l}
d \\
2
\end{array}\right) .
$$

(ii) Now consider the hyperplanes formed by $\boldsymbol{x}$ and any $d-1$ vertices from $F_{i}$. There are $\left(\begin{array}{c}d \\ d-1\end{array}\right)=\left(\begin{array}{l}d \\ 1\end{array}\right)=d$ such choices, and none of these intersect $S\left[\boldsymbol{X}_{1}, \ldots, \boldsymbol{X}_{d+1}\right]^{\circ}$.

(iii) Now the total number of hyperplanes formed by $\boldsymbol{x}$ and any $d-1$ vertices from $S\left[\boldsymbol{X}_{1}, \ldots, \boldsymbol{X}_{d+1}\right]$ is $\left(\begin{array}{l}d+1 \\ d-1\end{array}\right)$. Since $\left(\begin{array}{l}d+1 \\ d-1\end{array}\right)=\left(\begin{array}{c}d+1 \\ 2\end{array}\right)=\left(\begin{array}{l}d \\ 2\end{array}\right)+\left(\begin{array}{l}d \\ 1\end{array}\right)$, we have accounted for all hyperplanes in (i) and (i) above, and it follows from (12), taken for each $1 \leq i \leq d$, and from the disjointness of the $A_{i}$, that

$$
\alpha\left(\boldsymbol{x}, \boldsymbol{X}_{1}, \ldots, \boldsymbol{X}_{d+1}\right)=\left(\begin{array}{l}
d \\
2
\end{array}\right), \boldsymbol{x} \in \bigcup_{i=1}^{d+1} A_{i} .
$$

See Figure 2 for illustration of $\boldsymbol{x} \in \bigcup_{i=1}^{d+1} A_{i}$ and of cases (i) and (ii) above, for $d=3$.

Case 2(b): $\boldsymbol{x} \notin \bigcup_{i=1}^{d+1} A_{i}$.

Then $\boldsymbol{x} \in B_{i j}$ for at least one pair $\{i, j\}$, where $1 \leq i<j \leq d+1$. (Note that the $\left\{B_{i j}\right\}$ are not all mutually exclusive.) Thus $\boldsymbol{x}$ can "see" both of $F_{i}$ and $F_{j}$ and is in the "corner wedge" formed by $H\left(F_{i}\right)$ and $H\left(F_{j}\right)$. Now there are exactly two vertices, say $\boldsymbol{v}_{i j}^{(1)}$ and $\boldsymbol{v}_{i j}^{(2)}$, of $S\left[\boldsymbol{X}_{1}, \ldots, \boldsymbol{X}_{d+1}\right]$ that are not common to $F_{i}$ and $F_{j}$. Clearly, $\boldsymbol{x}$ can "see" both of these, too, but not via lines through the faces.

(i) The hyperplane formed by $\boldsymbol{x}$ with the $d-1$ vertices common to $F_{i}$ and $F_{j}$ separates $\boldsymbol{v}_{i j}^{(1)}$ and $\boldsymbol{v}_{i j}^{(2)}$, and the corresponding increment to $\alpha\left(\boldsymbol{x}, \boldsymbol{X}_{1}, \ldots, \boldsymbol{X}_{d+1}\right)$ is

$$
\text { increment }=1 \text {. }
$$

(ii) Now consider the $\left(\begin{array}{c}d-1 \\ 2\end{array}\right)$ hyperplanes formed by $\boldsymbol{x}, \boldsymbol{v}_{i j}^{(1)}, \boldsymbol{v}_{i j}^{(2)}$, and $d-3$ other vertices of $S\left[\boldsymbol{X}_{1}, \ldots, \boldsymbol{X}_{d+1}\right]$. (a) Suppose that at least one such hyperplane separates the remaining 
two vertices. Then, for each such hyperplane, none of the corresponding four hyperplanes which are formed from that hyperplane by substituting one of the two still available vertices for exactly one of $\boldsymbol{v}_{i j}^{(1)}$ and $\boldsymbol{v}_{i j}^{(2)}$ separates the remaining two vertices of $S\left[\boldsymbol{X}_{1}, \ldots, \boldsymbol{X}_{d+1}\right]$. Therefore, in this scenario involving the hyperplanes that use one or both of $\boldsymbol{v}_{i j}^{(1)}$ and $\boldsymbol{v}_{i j}^{(2)}$, the increment to $\alpha\left(\boldsymbol{x}, \boldsymbol{X}_{1}, \ldots, \boldsymbol{X}_{d+1}\right)$ satisfies

$$
1 \leq \text { increment } \leq\left(\begin{array}{c}
d-1 \\
2
\end{array}\right) \quad(=1 \text { if } d=3) .
$$

(b) On the other hand, if none of the $\left(\begin{array}{c}d-1 \\ 2\end{array}\right)$ hyperplanes using $\boldsymbol{x}$ with both of $\boldsymbol{v}_{i j}^{(1)}$ and $\boldsymbol{v}_{i j}^{(2)}$ separates the remaining two vertices, then, for each such hyperplane, exactly half of the four corresponding hyperplanes formed by substituting for exactly one of $\boldsymbol{v}_{i j}^{(1)}$ and $\boldsymbol{v}_{i j}^{(2)}$ do separate their remaining two vertices, depending on whether $\boldsymbol{x}$ is "high" or "low" in its wedge, relative to $S\left[\boldsymbol{X}_{1}, \ldots, \boldsymbol{X}_{d+1}\right]$. In all, there are $2(d-1)$ distinct hyperplanes formed by $\boldsymbol{x}$, exactly one of $\boldsymbol{v}_{i j}^{(1)}$ and $\boldsymbol{v}_{i j}^{(2)}$, and $d-2$ other vertices. Therefore, in this second scenario involving the hyperplanes that use one or both of $\boldsymbol{v}_{i j}^{(1)}$ and $\boldsymbol{v}_{i j}^{(2)}$, the increment to $\alpha\left(\boldsymbol{x}, \boldsymbol{X}_{1}, \ldots, \boldsymbol{X}_{d+1}\right)$ is one half of their number, i.e.,

$$
\text { increment }=d-1 \text {. }
$$

(c) Combining the above, it follows that, for $\boldsymbol{x} \in B_{i j}, \alpha\left(\boldsymbol{x}, \boldsymbol{X}_{1}, \ldots, \boldsymbol{X}_{d+1}\right)$ is given by the increment 1 in (14) plus an increment corresponding to either (15) or (16). In the first case this yields $2 \leq \alpha\left(\boldsymbol{x}, \boldsymbol{X}_{1}, \ldots, \boldsymbol{X}_{d+1}\right) \leq 1+\left(\begin{array}{c}d-1 \\ 2\end{array}\right)$, and in the second case it yields $\alpha\left(\boldsymbol{x}, \boldsymbol{X}_{1}, \ldots, \boldsymbol{X}_{d+1}\right)=1+(d-1)=d$. Thus we arrive at

$$
2 \leq \alpha\left(\boldsymbol{x}, \boldsymbol{X}_{1}, \ldots, \boldsymbol{X}_{d+1}\right) \leq \max \left\{d, 1+\left(\begin{array}{c}
d-1 \\
2
\end{array}\right)\right\}, \boldsymbol{x} \notin \bigcup_{i=1}^{d} A_{i} .
$$

See Figure 3 for illustration of $\boldsymbol{x} \in B_{i j}$ and cases (i) and (ii) above, and cases of $\boldsymbol{x}$ attaining the lower and upper bounds in (17), for $d=3$.

Completion of Case 2. Combining (13) and (17), and noting that we have

$$
2 \leq \alpha\left(\boldsymbol{x}, \boldsymbol{X}_{1}, \ldots, \boldsymbol{X}_{d+1}\right) \leq\left(\begin{array}{l}
d \\
2
\end{array}\right), \boldsymbol{x} \notin S\left[\boldsymbol{X}_{1}, \ldots, \boldsymbol{X}_{d+1}\right]^{\circ}
$$

Now, for any subset $\left\{\boldsymbol{X}_{i_{1}}, \ldots, \boldsymbol{X}_{i_{d+1}}\right\}$, the analogue of (9) is

$$
\begin{aligned}
& h_{I}\left(\boldsymbol{x}, \boldsymbol{X}_{i_{1}}, \ldots, \boldsymbol{X}_{i_{d+1}}\right)= \\
& \quad \mathbf{1}\left\{h_{S}\left(\boldsymbol{x}, \boldsymbol{X}_{i_{1}}, \ldots, \boldsymbol{X}_{i_{d+1}}\right)=1\right\}+\frac{\alpha\left(\boldsymbol{x}, \boldsymbol{X}_{i_{1}}, \ldots, \boldsymbol{X}_{i_{d+1}}\right)}{\left(\begin{array}{c}
d+1 \\
2
\end{array}\right)} \mathbf{1}\left\{h_{S}\left(\boldsymbol{x}, \boldsymbol{X}_{i_{1}}, \ldots, \boldsymbol{X}_{i_{d+1}}\right)=0\right\},
\end{aligned}
$$

subject to $\boldsymbol{x} \notin H\left(\mathbb{X}_{n}\right)$. We now introduce the average $\alpha\left(\boldsymbol{x}, \mathbb{X}_{n}\right)$ of $\alpha\left(\boldsymbol{x} ; \boldsymbol{X}_{i_{1}}, \ldots, \boldsymbol{X}_{i_{d+1}}\right)$ over the cases with $\boldsymbol{x} \notin S\left[\boldsymbol{X}_{1}, \ldots, \boldsymbol{X}_{d+1}\right]$ (i.e., with $\left.h_{S}=0\right)$, which number $\left(\begin{array}{c}n \\ d+1\end{array}\right)\left(1-D_{S}(\boldsymbol{x})\right)$. Thus

$$
\alpha\left(\boldsymbol{x}, \mathbb{X}_{n}\right)=\left[\left(\begin{array}{c}
n \\
d+1
\end{array}\right)\left(1-D_{S}(\boldsymbol{x})\right)\right]^{-1} \sum_{h_{S}=0} \alpha\left(\boldsymbol{x} ; \boldsymbol{X}_{i_{1}}, \ldots, \boldsymbol{X}_{i_{d+1}}\right) .
$$

Now averaging over (19), we obtain (in abbreviated notation)

$$
\begin{aligned}
D_{I}(\boldsymbol{x}) & =\left(\begin{array}{c}
n \\
d+1
\end{array}\right)^{-1}\left[\sum_{h_{S}=1} 1+\sum_{h_{S}=0} \frac{\alpha\left(\boldsymbol{x} ; \boldsymbol{X}_{i_{1}}, \ldots, \boldsymbol{X}_{i_{d+1}}\right)}{\left(\begin{array}{c}
d+1 \\
2
\end{array}\right)}\right] \\
& =\left(\begin{array}{c}
n \\
d+1
\end{array}\right)^{-1}\left[\left(\begin{array}{c}
n \\
d+1
\end{array}\right) D_{S}(\boldsymbol{x})+\left(\begin{array}{c}
n \\
d+1
\end{array}\right)\left(1-D_{S}(\boldsymbol{x})\right) \frac{\alpha\left(\boldsymbol{x} ; \mathbb{X}_{n}\right)}{\left(\begin{array}{c}
d+1 \\
2
\end{array}\right)}\right],
\end{aligned}
$$


and we have established (10) and (11), completing the proof of Theorem 2.

Remark 3. (i) For $\boldsymbol{x} \in S\left[\boldsymbol{X}_{i_{1}}, \ldots, \boldsymbol{X}_{i_{d+1}}\right]^{\circ}$, it is immediate from the proofs of Theorems 1 and 2 that, for every dimension $d \geq 2$, the simplicial and interdirections "kernels" evaluated at $\left\{\boldsymbol{X}_{i_{1}}, \ldots, \boldsymbol{X}_{i_{d+1}}\right\}$ agree.

(ii) For $\boldsymbol{x} \notin S\left[\boldsymbol{X}_{i_{1}}, \ldots, \boldsymbol{X}_{i_{d+1}}\right]^{\circ}$, and for $d \geq 3$, it is seen from the proof of Theorem 2 that $\alpha\left(\boldsymbol{x} ; \boldsymbol{X}_{i_{1}}, \ldots, \boldsymbol{X}_{i_{d+1}}\right)$ attains the upper bound $\left(\begin{array}{l}d \\ 2\end{array}\right)$ for all $\boldsymbol{x}$ such that for some face of $S\left[\boldsymbol{X}_{i_{1}}, \ldots, \boldsymbol{X}_{i_{d+1}}\right]$ the line from $\boldsymbol{x}$ to the vertex of $S\left[\boldsymbol{X}_{i_{1}}, \ldots, \boldsymbol{X}_{i_{d+1}}\right]$ not included in that face passes through the face, and otherwise satisfies the lower upper bound $\max \left\{d, 1+\left(\begin{array}{c}d-1 \\ 2\end{array}\right)\right\}$, except that these bounds agree in the case $d=3$.

(iii) For $d=2$, the simplicial and interdirections depths are equivalent, as shown by Theorem 1, and the simplicial depth is then preferred on the basis of its significantly lesser computational burden of $O(n \log n)$ versus $O\left(n^{3}\right)$.

(iv) For $d=3$, for $\boldsymbol{x} \notin S\left[\boldsymbol{X}_{i_{1}}, \ldots, \boldsymbol{X}_{i_{4}}\right]^{\circ}$ the interdirections indicator kernel assigns one of two possible values, $1 / 3$ and 1/2. Therefore, the average $\alpha\left(\boldsymbol{x}, \mathbb{X}_{n}\right) /\left(\begin{array}{c}d+1 \\ 2\end{array}\right)$ in (10) lies between these values, showing that the simplicial and interdirections depths perform very similarly. Again, therefore, the simplicial depth is preferred on the basis of its more favorable computational burden of $O\left(n^{2}\right)$ versus $O\left(n^{4}\right)$.

(v) For $d=4$, the average $\alpha\left(\boldsymbol{x}, \mathbb{X}_{n}\right) /\left(\begin{array}{c}d+1 \\ 2\end{array}\right)$ in (10) lies between $1 / 5$ and $3 / 5$, indicating again a rather close correspondence between the two depths. Again, therefore, the simplicial depth is preferred on the basis of its computational burden of $O\left(n^{4}\right)$ versus $O\left(n^{5}\right)$.

(vi) For $d \geq 5$, the number of possible values assigned to nonmembership increases slowly with $d$, with

$$
\frac{4}{d(d+1)} \leq \alpha\left(\boldsymbol{x}, \mathbb{X}_{n}\right) /\left(\begin{array}{c}
d+1 \\
2
\end{array}\right) \leq \frac{d-1}{d+1}
$$

Again, they perform rather similarly. Although the computational burden is now $O\left(n^{d+1}\right)$ for each, the constant in this order is more favorable for the simplicial depth, which also might be preferred on the basis of greater conceptual appeal.

\subsection{A simulation study comparing computation times of $D_{I}$ and $D_{S}$}

A small simulation study was carried out to compare computation times of $D_{I}$ and $D_{S}$ in the most significant case of dimension $d=2$. For $F$ standard bivariate normal, 1000 samples were generated for each of sample sizes $n=50$ and 100. For each sample, each of $D_{I}$ and $D_{S}$ was evaluated for 5 points in $\mathbb{R}^{2}$, corresponding to the origin $(0,0)$ and the points of form $(x, x)$ on the $50 \%, 75 \%, 90 \%$, and $99 \%$ quantile contours of $F$. The corresponding computation times averaged over all 1000 samples are reported in Table $\mathbf{1}$.

For $D_{I}\left(\boldsymbol{x}, \mathbb{X}_{n}\right)$, computation was carried out using $(\mathrm{A})$ : the naive algorithm of order $O\left(n^{3}\right)$, which we coded in $\mathrm{R}$. For $D_{S}\left(\boldsymbol{x}, \mathbb{X}_{n}\right)$, computation was carried out for each of four implementations:

(B): the $O(n \log n)$ algorithm of Rousseeuw and Ruts (1996), which we coded in R;

(C): the naive algorithm of order $O\left(n^{3}\right)$, which we coded in R;

(D): the naive algorithm of order $O\left(n^{3}\right)$ available in Fortran in the R package ddalpha;

(E): the naive algorithm of order $O\left(n^{3}\right)$ available in $\mathrm{C}$ in the $\mathrm{R}$ package localdepth. 


\begin{tabular}{|r|rr|rr|rr|rr|rr|}
\hline & \multicolumn{2}{|c|}{$A$} & \multicolumn{2}{|c|}{$B$} & \multicolumn{2}{|c|}{$C$} & \multicolumn{2}{|c|}{$D$} & \multicolumn{2}{|c|}{$E$} \\
\cline { 2 - 10 }$n$ & Mean & SD & Mean & SD & Mean & SD & Mean & SD & Mean & $S D$ \\
\hline 50 & 17.5 & 0.4 & 0.015 & 0.002 & 2.9 & 0.1 & 0.01 & 0.01 & 0.01 & 0.02 \\
100 & 140.2 & 0.8 & 0.030 & 0.002 & 23.6 & 0.4 & 0.10 & 0.02 & 0.07 & 0.01 \\
\hline
\end{tabular}

Table 1: Computation times (in sec., mean and SD) for interdirections depth (algorithm A) and simplicial depth (algorithms B-E), at 5 selected points of standard bivariate normal, for 1000 samples each of sizes $n$ $=50$ and 100 .

The findings in Table 1 support the following conclusions:

- The relative efficiency of $D_{S}$ over $D_{I}$ is over 1000-fold at sample size 50 and over 4000-fold at sample size 100, as seen by comparing times for algorithms A and B. This improvement with increasing sample size follows the rate $O\left(n^{2} / \log n\right)$, from comparing $O\left(n^{3}\right)$ with $O(n \log n)$.

- As expected, doubling the sample size from 50 to 100 increases computation time by only a factor of 2 for the efficient algorithm B of order $O(n \log n)$, but by a factor of 8 for the naive algorithms A, C, D, and $\mathrm{E}$ of order $O\left(n^{3}\right)$.

- The computational burden of $D_{I}$ is about 6 times that of $D_{S}$, as seen by comparing times for the naive algorithms $\mathrm{A}$ and $\mathrm{C}$, each coded in the same language. Whereas $D_{S}$ evaluates a single indicator function for each of the $\left(\begin{array}{l}n \\ 3\end{array}\right)$ triplets from the sample, $D_{I}$ evaluates and averages 3 indicator functions for each triplet.

- Coding algorithm B in either Fortran or C language should improve computation time by about a factor of 300, as seen by comparing times for algorithms C, D, and E.

- Analogous simulations for dimensions $d \geq 3$ would yield similar perspectives.

\section{Applications of the connection between $D_{I}$ and $D_{S}$}

Theorems 1 and 2, Remark 3, and the findings from Table 1 altogether suggest replacing interdirections approaches by simplicial depth approaches. The simplicial depth performs comparably to the interdirections depth in efficiency while offering some computational advantage as well as greater conceptual appeal. Of course, for dimension $d \geq 5$, both depths are prohibitive computationally, except for very small sample sizes. However, especially for $d=2$ and also for $d=3$ and 4 , the computational advantage of the simplicial depth is substantial. On this basis, various statistical procedures involving interdirections may profitably be modified by substituting use of the simplicial depth. Multivariate sign tests for location are discussed in Section 5.1, and several other possibilities are noted in Section 5.2 .

\subsection{Multivariate sign tests for location}

We discuss five multivariate sign tests for location.

1. A key benchmark in extending the univariate sign test to higher dimension is the bivariate sign test $B_{n}$ invented by Blumen (1958) for testing $H_{0}: \boldsymbol{\theta}=\boldsymbol{\theta}_{0}$, with $\boldsymbol{\theta}$ defined as a very general notion of median (equivalent to the halfspace median defined by Zuo and Serfling, 2000b) which generalizes the point of angular symmetry. The test statistic $B_{n}$ is affine invariant and asymptotically $\chi_{2}^{2}$ in distribution. It is locally optimal against normal (and all elliptical) alternatives, with ARE $\pi / 4=0.785$ relative to the bivariate Hotelling test in the normal case. 
2. Also, again for the bivariate case, and for testing central symmetry about $\boldsymbol{\theta}_{0}$, Oja and Nyblom (1989) formulate and study a class of sign test statistics which includes $B_{n}$ as well as other statistics of special interest, including a statistic $U_{n}$ which equivalently to $B_{n}$ is locally optimal against normal (and all elliptical) alternatives but improves upon $B_{n}$ against certain nonelliptical, but centrally symmetric, alternatives such as bivariate Laplace.

3. The above-mentioned Liu (1990) test $S_{n}$, available in any dimension $d$, is readily seen in the bivariate case to be equivalent to $U_{n}$ :

$$
S_{n}=\frac{1}{4}-D_{n}\left(\boldsymbol{\theta}_{0}, \mathbb{X}_{n}\right)=\frac{3}{n(n-1)(n-2)} U_{n}+\frac{-3 n}{4(n-1)(n-2)} .
$$

Therefore, applying the limit distribution for $U_{n}$ given in Example 5.1 of Oja and Nyblom (1989), we obtain for $S_{n}$ a weighted sum of chi-square variates as its limit distribution:

$$
\frac{4}{3} n S_{n}+1 \stackrel{d}{\longrightarrow} \frac{4}{\pi^{2}} \sum_{k=1}^{\infty}(2 k-1)^{-2} \chi_{k}^{2}(2),
$$

where the $\chi_{k}^{2}(2)$ are independent chi-square variates with 2 degrees of freedom. The equivalence with $U_{n}$ yields for $S_{n}$ the ARE $\pi / 4=0.785$ relative to the bivariate Hotelling test in the normal case, the same as for $B_{n}$. (This agrees with the aforementioned ARE of the simplicial median versus the mean. However, the equivalent simplicial median test being computationally much more intensive, the use of $S_{n}$ is much preferred.)

4. As discussed in Section 3, Randles (1989) introduces the interdirections in formulating a new sign test statistic $V_{n}$ for testing $H_{0}: \boldsymbol{\theta}=\boldsymbol{\theta}_{0}$ in the setting of a sample $\mathbb{X}_{n}$ in $\mathbb{R}^{d}$, for any dimension $d \geq 2$, from a distribution angularly symmetric about $\boldsymbol{\theta}$. However, its asymptotic distribution is derived in general only for $F$ having elliptical directions. For $d=2, V_{n}$ reduces to $B_{n}$. Although a preprint of Liu (1990) is cited, with reference to both its definition of angular symmetry and its proposed invariant test of symmetry using simplicial depth, the direct connections between simplicial depth and interdirections are not noted.

5. Chaudhuri and Sengupta (1993) introduce a general form of sign test statistic $W_{n}$ for any dimension $d$ and note that (i) $W_{n}$ includes Randles' $V_{n}$ as a special case, and (ii) for $d=2, W_{n}$ is equivalent both to Oja and Nyblom's $U_{n}$ and to the simplicial depth of $\boldsymbol{\theta}_{0}$ (and thus to $S_{n}$ ). They comment that analogues of (ii) do not hold for $d \geq 3$, and that the implications and consequences of these "intriguing" connections "are not fully understood at present".

Thus we see that, via the direct connection between simplicial depth and interdirections, the statistics $S_{n}$ and $V_{n}$ are closely related and nearly equivalent for any dimension $d$. Also, for $d=2$ the statistics $S_{n}, U_{n}, V_{n}$, and $W_{n}$ are all essentially equivalent, at least asymptotically.

The equivalence in performance of $S_{n}$ and $V_{n}$ in the bivariate case is corroborated by a small simulation study also comparing with the Hotelling $T^{2}$ test and including computation times. For each of $F$ bivariate $N(\mathbf{0}, \boldsymbol{I}), N(\boldsymbol{\Delta}, \boldsymbol{I}), N(2 \boldsymbol{\Delta}, \boldsymbol{I})$, and $N(3 \boldsymbol{\Delta}, \boldsymbol{I}), 10,000$ samples were generated for each of sample sizes $n=50$ and 100. Here $\boldsymbol{\Delta}=0.0815(1,1)$ for $n=50$ and $=0.0567(1,1)$ for $n=100$, so that the Hotelling $T^{2}$ test at level 0.05 has power 0.10 at $N(\boldsymbol{\Delta}, \boldsymbol{I})$, based on the relevant central and noncentral $F_{2,48}$ and $F_{2,98}$ distributions. For $V_{n}$ the cutoff point for a level 0.05 test is the 0.95 quantile of the limit chi-square distribution with 2 degrees of freedom, whereas for $S_{n}$ via the statistic $\frac{4}{3} n S_{n}+1$ the cutoff point is 1.218 based on the limit distribution in (20). For each distribution and each test statistic, the empirical rejection rate and mean computation time, based on 10,000 samples, are shown in Table 2. Computations are coded in R, using for $S_{n}$ the efficient $O(n \log n)$ algorithm. 


\begin{tabular}{|c|c|c|c|c|c|c|c|}
\hline \multirow[b]{2}{*}{$n$} & \multirow[b]{2}{*}{ Shift } & \multicolumn{2}{|r|}{$T^{2}$} & \multicolumn{2}{|r|}{$\overline{S_{n}}$} & \multicolumn{2}{|r|}{$\overline{V_{n}}$} \\
\hline & & Rate & Time & Rate & Time & Rate & Time \\
\hline \multirow[t]{4}{*}{50} & $\overline{0}$ & 0.048 & $0.0002(.0005)$ & 0.055 & $0.003(0.001)$ & 0.049 & $4.29(0.08)$ \\
\hline & $\Delta$ & 0.095 & $0.0002(.0004)$ & 0.096 & $0.003(0.001)$ & 0.087 & $4.30(0.09)$ \\
\hline & $2 \Delta$ & 0.267 & $0.0002(.0005)$ & 0.223 & $0.003(0.001)$ & 0.216 & $4.28(0.08)$ \\
\hline & $3 \Delta$ & 0.544 & $0.0002(.0004)$ & 0.463 & $0.003(0.001)$ & 0.445 & $4.30(0.09)$ \\
\hline \multirow[t]{4}{*}{100} & 0 & 0.052 & $0.0002(.0004)$ & 0.054 & $0.007(0.001)$ & 0.052 & $25.3(0.2)$ \\
\hline & $\Delta$ & 0.099 & $0.0002(.0004)$ & 0.096 & $0.007(0.001)$ & 0.091 & $25.2(0.3)$ \\
\hline & $2 \Delta$ & 0.272 & $0.0002(.0004)$ & 0.225 & $0.007(0.001)$ & 0.219 & $25.3(0.2)$ \\
\hline & $3 \Delta$ & 0.546 & $0.0002(.0005)$ & 0.459 & $0.007(0.001)$ & 0.451 & $25.7(0.2)$ \\
\hline
\end{tabular}

Table 2: Rejection rates and computation times (in sec., mean and SD) for Hotelling's $T^{2}$, Liu's $S_{n}$, and Randles' $V_{n}$ tests of location at level 0.05 , for 10,000 samples each of sizes $n=50$ and 100 from the bivariate standard normal distribution and three location shifts of that distribution. The SDs of the observed rejection rates are $0.002,0.003,0.004$, and 0.005 for shifts $0, \boldsymbol{\Delta}, 2 \boldsymbol{\Delta}$, and $3 \boldsymbol{\Delta}$, respectively.

The findings in Table 2 support the following conclusions, within negligible statistical error:

- With respect to Type I error, the three procedures perform reliably and equivalently.

- With respect to Type II error, the $S_{n}$ and $V_{n}$ tests perform equivalently and with quite high relative efficiency with respect to the $T^{2}$ test (even greater than the asymptotic $\mathrm{RE}$ of 0.785$)$.

- However, with respect to computation time (for $d=2$ ), $S_{n}$ is dramatically superior to $V_{n}$ by factors of approximately 1400 and 3600 for $n=50$ and 100, respectively.

On the basis of the above considerations, for testing a center of symmetry by a sign test, the simplicial depth approach using $S_{n}$ is simple conceptually, relatively attractive computationally, competitively efficient, and accompanied by asymptotic distribution theory under merely the angular symmetry assumption. Further, it is affine invariant. This sign test is worthy of more extensive investigation, not only for the bivariate case but also, through some variants, for higher dimension. It evidently captures in an efficient way information about location.

\subsection{Further examples}

We note several other statistical procedures based on interdirections that are candidates for developing modifications based on simplicial depth to improve computational time while maintaining the same or comparable efficiency. Peters and Randles (1990) develop a type of signed-rank test for location based on the interdirections. Jan and Randles (1994) develop a signed-sum test of multivariate location using interdirections, and Randles and Peters (1990) and Um and Randles (1998) extend it to the multi-sample setting. Asymptotically locally optimal tests of multivariate location in the setting of elliptical symmetry using interdirections are developed by Hallin and Paindaveine (2002b). Similarly, Hallin and Paindaveine (2002c) construct interdirections-based tests against serial dependence, and Hallin and Paindaveine (2005) provide tests for linear models with VARMA errors. These various possibilities for modification using simplicial depth will be explored in separate studies. 


\section{Acknowledgments}

The first author gratefully acknowledges encouragement from G. L. Thompson. Both authors thank Marc Hallin, an Associate Editor, and two anonymous reviewers for very constructive suggestions. Also, support under National Science Foundation Grant DMS1106691 is sincerely acknowledged.

\section{References}

[1] Aloupis, G., Cortes, C., Gomez, F., Soss, M. and Toussaint, G. (2002). Lower bounds for computing statistical depth. Computational Statistics $\& 3$ Data Analysis 40 223-229.

[2] Aloupis, G., Langerman, S., Soss, M., and Toussaint, G. Algorithms for bivariate medians and a Fermat-Torricelli problem for lines. Computational Geometry: Theory and Applications 26 69-79.

[3] Arcones, M. A., Chen, Z. and Giné, E. (1994). Estimators related to U-processes with applications to multivariate medians: asymptotic normality. Annals of Statistics 22 $1460-1477$.

[4] Arcones, M. A. and Giné, E. (1993). Limit theorems for $U$-processes. Annals of Probability 21 1494-1542.

[5] Blumen, I. (1958). A new bivariate sign test. Journal of the American Statistical Association 53 448-456.

[6] Boros, E. and Füredi, Z. (1984). The maximal number of covers by the triangles of a given vertex set on the plane. Geometriae Dedicata 1 69-77.

[7] Burr, M. A., Rafalin, E. and Souvaine, D. L. (2006). Simplicial depth: an improved definition, analysis, and efficiency. In Data Depth: Robust Multivariate Analysis, Computational Geometry and Applications (R. Y. Liu, R. Serfling, D. L. Souvaine, eds.), pp. 195-209. DIMACS Series in Discrete Mathematics and Theoretical Computer Science, Volume 72, American Mathematical Society, Providence, Rhode Island.

[8] Cheng, A. Y. and Ouyang, M. (2001). On algorithms for simplicial depth. In Proceedings of 13th Canadian Conference on Computational Geometry (CCCG'01), pp. 53-56.

[9] de la Peña, V. H. and Ginè, E. (1999). Decoupling: From Dependence to Independence. Springer.

[10] Dümbgen, L. (1992). Limit theorems for the simplicial depth. Statistics and Probability Letters 14 119-128.

[11] Gil, J., Steiger, W., and Wigderson, A. (1992). Geometric medians. Discrete Mathematics 108 37-51.

[12] Hallin, M. and Paindaveine, D. (2002a). Multivariate signed ranks: Randles's interdirections or Tyler's angles? In Statistical Data Analysis Based On the $L_{1}$-Norm and Related Methods (Y. Dodge, ed.), pp. 25-38. Birkhaüser.

[13] Hallin, M. and Paindaveine, D. (2002b). Optimal tests for multivariate location based on interdirections and pseudo-Mahalanobis ranks. Annals of Statistics 30 1103-1133. 
[14] Hallin, M. and Paindaveine, D. (2002c). Optimal procedures based on interdirections nd pseudo-Mahalanobis ranks for testing multivariate elliptical white noise against ARMA dependence. Bernoulli 8 787-815.

[15] Hallin, M. and Paindaveine, D. (2005). Affine-invariant aligned rank tests for the multivariate general linear model with VARMA errors. Journal of Multivariate Analysis $93122-163$.

[16] Jan, S.-L. and Randles, R. H. (1994). A multivariate signed-sum test for the one-sample location problem. Journal of Nonparametric Statistics 4 49-63.

[17] Khuller, S. and Mitchell, J. S. B. (1990). On a triangle counting problem. Information Processing Letters 33 319-321.

[18] Langerman, S. and Steiger, W. (2003). Optimization in arrangements. In Proceedings of the 20th International Symposium on Theoretical Aspects of Computer Science (STACS 2003) (H. Alt and M. Habib, eds.), pp. 50-61.

[19] Liu, R. Y. (1988). On a notion of simplicial depth. Proceedings of the National Academy of Science USA 85 1732-1734.

[20] Liu, R. Y. (1990). On a notion of data depth based on random simplices. Annals of Statistics 18 405-414.

[21] Liu, R. Y., Parelius, J. M. and Singh, K. (1999). Multivariate analysis by data depth: Descriptive statistics, graphics and inference (with discussion). Annals of Statistics $\mathbf{2 7}$ $783-858$.

[22] Oja, H. and Nyblom, J. (1989). Bivariate sign tests. Journal of the American Statistical Assocation 84 249-259.

[23] Peters, D. and Randles, R. H. (1990). A multivariate signed-rank test for the onesampled location problem. Journal of the American Statistical Association 85 552-557.

[24] Randles, R. H. (1989). A distribution-free multivariate sign test based on interdirections. Journal of the American Statistical Assocation 84 1045-1050.

[25] Randles, R. H. (2000). A simpler, affine-invariant, multivariate, distribution-free sign test. Journal of the American Statistical Assocation 95 1263-1268.

[26] Randles, R. H. and Peters, D. (1990). Multivariate rank tests for the two-sample location problem. Communications in Statistics: Theory and Methods 19 4225-4238.

[27] Rousseeuw, P. J. and Ruts, I. (1996). Algorithm AS 307: Bivariate location depth. Journal of the Royal Statistical Society Series C 45 516-526.

[28] Serfling, R. (2006a). Depth functions in nonparametric multivariate analysis. In Data Depth: Robust Multivariate Analysis, Computational Geometry and Applications (R. Y. Liu, R. Serfling, D. L. Souvaine, eds.), pp. 1-16. DIMACS Series in Discrete Mathematics and Theoretical Computer Science, Volume 72, American Mathematical Society, Providence, Rhode Island.

[29] Serfling, R. (2006b). Multivariate symmetry and asymmetry. In The Encyclopedia of Statistical Sciences, Second Edition, Vol. 8 (S. Kotz, N. Balakrishnan, C. B. Read, B. Vidakovic and N. L. Johnson, eds.), pp. 5338-5345. Wiley. 
[30] Serfling, R. (2010). Equivariance and invariance properties of multivariate quantile and related functions, and the role of standardization. Journal of Nonparametric Statistics 22 915-936.

[31] Tukey, J. W. (1975). Mathematics and the picturing of data. In Proceedings of the International Congress of Mathematicians, Vancouver 1974 (R. D. James, ed.), 2 523531.

[32] Um, Y. and Randles, R. H. (1998). Nonparametric tests for the multivariate multisample location problem. Statistica Sinica 8 801-812.

[33] Zuo, Y. and Serfling, R. (2000a). General notions of statistical depth function. Annals of Statistics 28 461-482.

[34] Zuo, Y. and Serfling, R. (2000b). On the performance of some robust nonparametric location measures relative to a general notion of multivariate symmetry. Journal of Statistical Planning and Inference 84 55-79. 


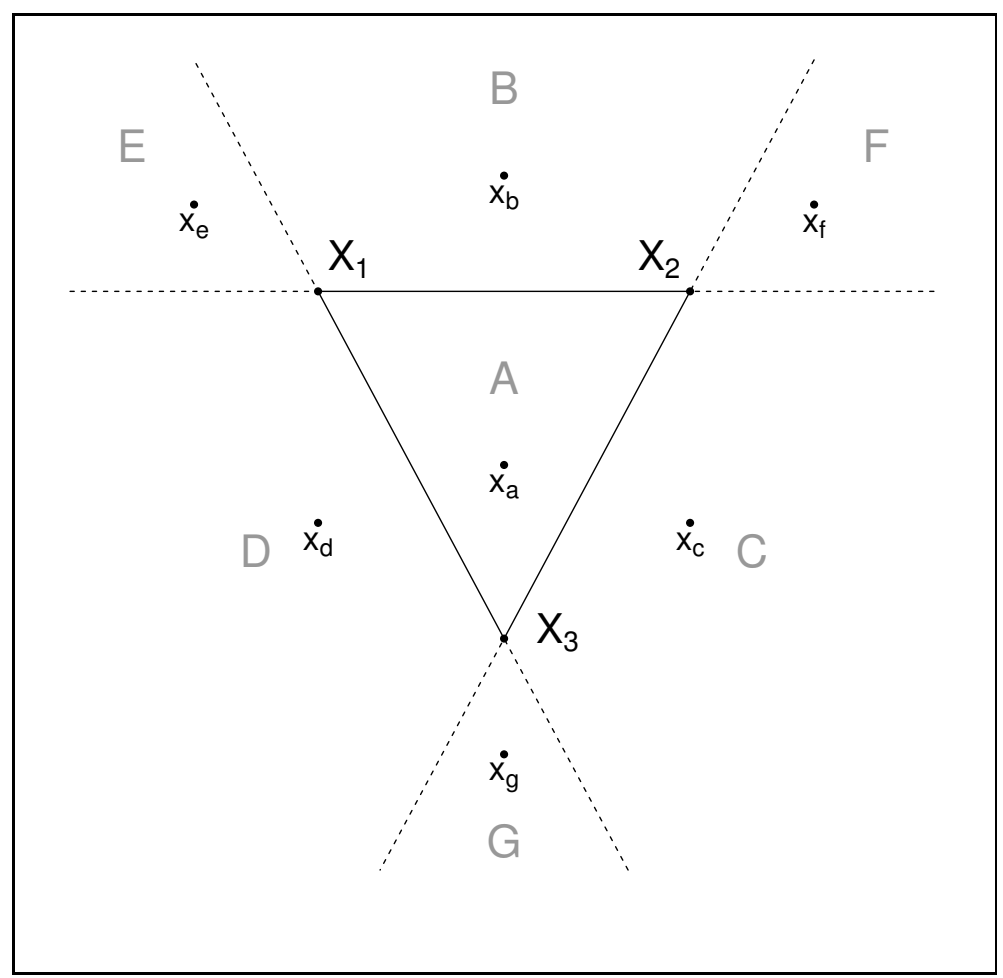

Figure 1: Illustration for Theorem 1. Trangular simplex $S\left[\boldsymbol{X}_{1} \boldsymbol{X}_{2} \boldsymbol{X}_{3}\right]$ in $\mathbb{R}^{2}$. Regions $A, B, C, D, E, F$, and $G$ with representative points $\boldsymbol{x}_{a}, \boldsymbol{x}_{b}, \boldsymbol{x}_{c}, \boldsymbol{x}_{d}, \boldsymbol{x}_{e}, \boldsymbol{x}_{f}$, and $\boldsymbol{x}_{g}$. For example, the point $\boldsymbol{x}_{b}$ lying in $B$ is normal to the face $\boldsymbol{X}_{1} \boldsymbol{X}_{2}$ and can "see" vertex $\boldsymbol{X}_{3}$. On the other hand, the point $\boldsymbol{x}_{e}$ lying in the "wedge" $E$ formed by the lines through faces $\boldsymbol{X}_{1} \boldsymbol{X}_{2}$ and $\boldsymbol{X}_{1} \boldsymbol{X}_{3}$ can also "see" $\boldsymbol{X}_{3}$ but not via lines through the simplex. 

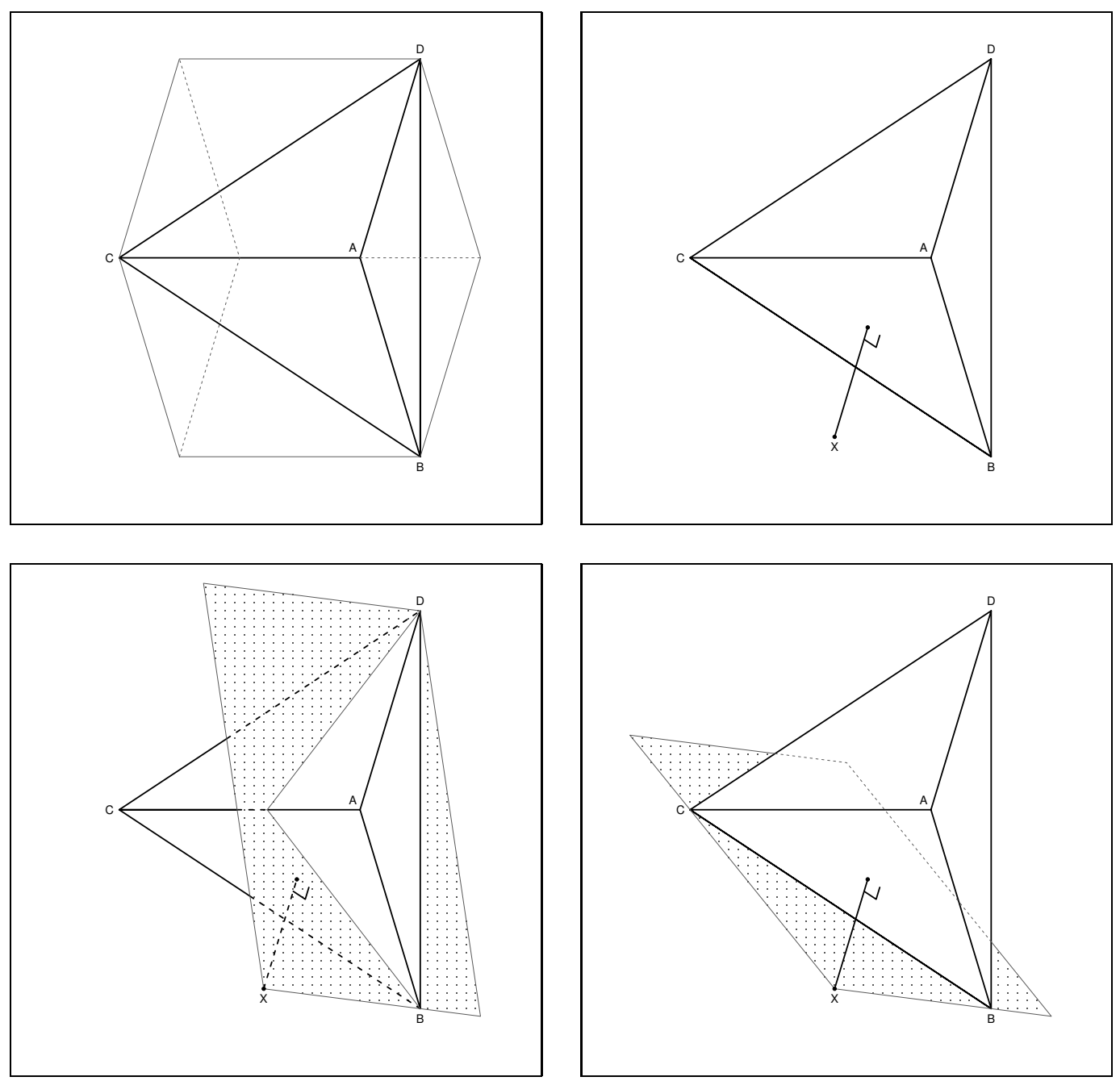

Figure 2: Illustration 1 for Theorem 2. Upper left: Tetrahedral simplex $S[A, B, C, D]$ embedded in unit cube in $\mathbb{R}^{3}$. Consider face ACD as "on top". Upper right: $X$ is normal to the triangle ABC and can "see" through $S[A, B, C, D]$ to vertex $\mathrm{D}$. There are six hyperplanes formed by $\mathrm{X}$ and any two of A, B, C, and D. Lower left: As shown, the hyperplane of XBD passes through $S[A, B, C, D]$ and separates A and C. Similarly, the hyperplane of XCD separates A and B, and the hyperplane of XAD separates B and C. Lower right: As shown, the hyperplane of XBC intersects $S[A, B, C, D]$ only on the edge $\mathrm{BC}$ and does not separate A and D. Similarly, the hyperplane of XAB does not separate C and D, nor does that of XAC separate B and D. 

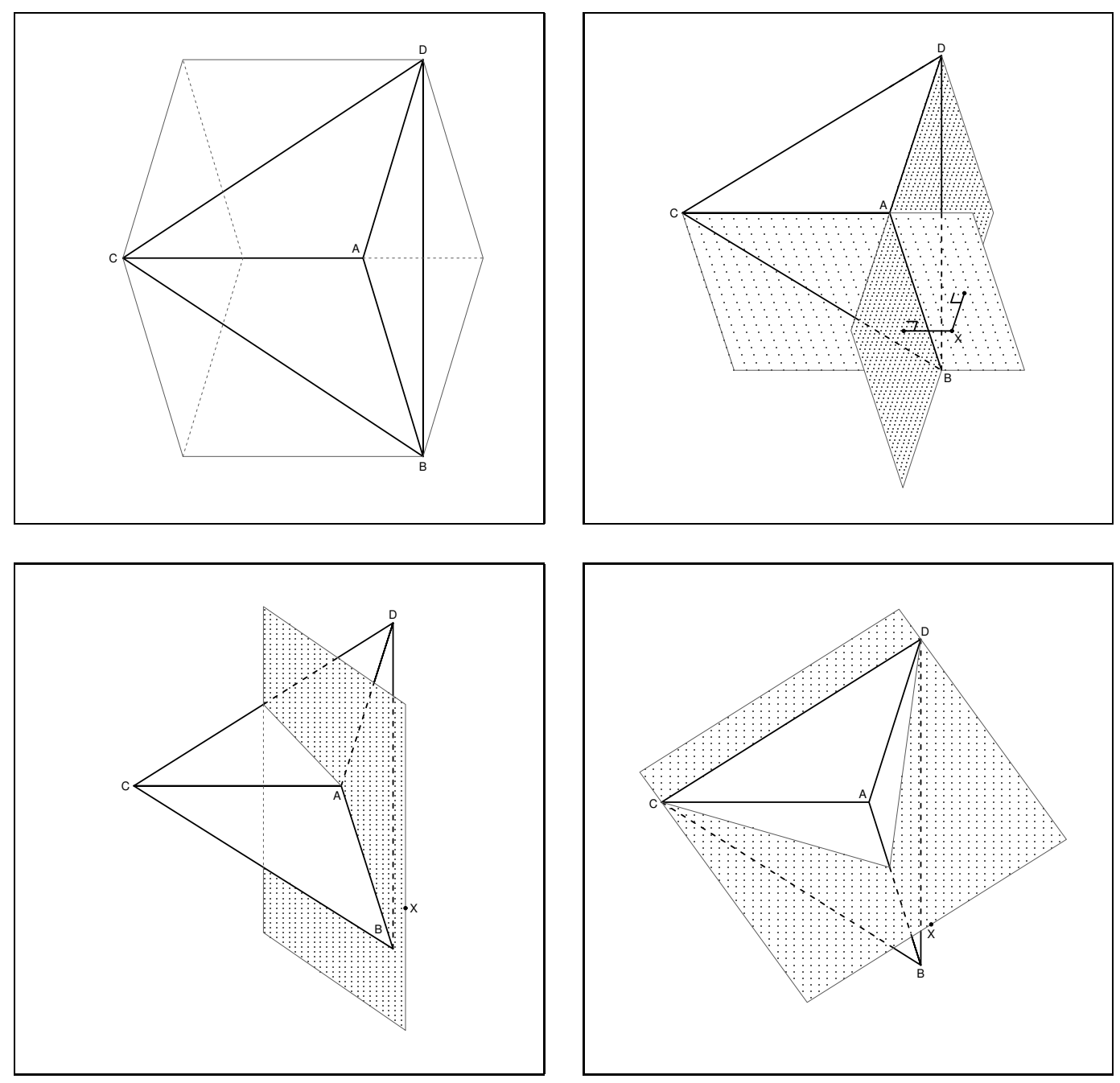

Figure 3: Illustration 2 for Theorem 2. Upper left: Tetrahedral simplex $S[A, B, C, D]$ embedded in unit cube in $\mathbb{R}^{3}$. Consider face ACD as "on top". Upper right: $X$ is in the wedge formed by the hyperplane of $\mathrm{ABC}$ and the hyperplane of $\mathrm{ABD}$ and is normal to each. There are six hyperplanes formed by $\mathrm{X}$ and any two of A, B, C, and D. Lower left: The hyperplane of XAB passes through $S[A, B, C, D]$ and separates $\mathrm{C}$ and $\mathrm{D}$, regardless of the "height" of X in the wedge. Lower right: The hyperplanes of XAC, XAD, XBC, XBD, and XCD do or do not intersect the interior of $S[A, B, C, D]$, depending on the "height" of X in the wedge. In particular, for X having "height" as shown, the hyperplane of XCD intersects $S[A, B, C, D]$ and separates $\mathrm{A}$ and $\mathrm{B}$, while those of $\mathrm{XAC}, \mathrm{XAD}, \mathrm{XBC}$, and XBD do not intersect the interior of $S[A, B, C, D]$, yielding altogether just 1 intersection and separation. On the other hand, for X with sufficiently higher "height", the hyperplanes of both XAC and XAD intersect the interior of $S[A, B, C, D]$ and separate $\mathrm{B}$ and $\mathrm{D}$, and $\mathrm{B}$ and $\mathrm{C}$, respectively, while those of XBC, XBD, and XCD do not intersect the interior of $S[A, B, C, D]$, yielding altogether 2 intersections and separations. A similar count holds for X sufficiently low in "height". 